\title{
EL MOTIVO DEL ENTIERRO EN LA BALADA ROMÁNICA
}

Aurelio Gonzćlez

Este trabajo no pretende agotar, ni mucho menos, las posibilidades de estudio que puede tener un motivo como el del entierro en un campo tan amplio como lo es el de la llamada balada europea; solamente es una cala que busca señalar cuáles podrían ser algunos de los puntos más interesantes en el funcionamiento de un mismo motivo en distintas tradiciones y temas baladísticos.

Para hacer esta cala se ha seleccionado un corpus a partir de una muestra antológica, representativa de las distintas tradiciones románicas. Las tradiciones estudiadas son las siguientes: portuguesa (Madeira), española (Asturias y Andalucía), catalana, occitana, francesa e italiana (Piamonte). ${ }^{1}$

Como lo que se busca es señalar líneas generales y no el detalle, cuando en las antologías seleccionadas aparece más de una versión del tema baladístico que contiene el motivo del entierro se ha escogido la más completa (este caso sólo se presenta en las antologias portuguesa e italiana). La selección de antologías no ha sido arbitraria ya que todas ellas, por la seriedad de sus autores y la calidad del material incluido, son representativas de las distintas tradiciones y permiten ver cuáles son las líneas generales que rigen la presencia y el funcionamiento del motivo del entierro. Las diferencias se pueden estudiar en un nivel general, comparativo en la misma tradición (en diferentes temas) o entre distintas tradiciones.

Una vez revisado el material antes menoionado se encontraron $\mathbf{4 0}$ textos que contienen el motivo del entierro (4 portugueses, 10 españoles, 5 catalanes, 3 occitanos, 8 franceses y 10 italianos), correspondientes a 10 temas baladísticos distintos: ${ }^{2}$ señas del esposo, doncella raptada, muerte al retorno de la ausencia, amor más poderoso que la muerte, muerte fingida, rescate del objeto perdido, muerte en la guerra, la amada difunta, venganza de la doncella seducida y muerte por mal de amores. ${ }^{3}$

1 Para las colecciones utilizadas véase la bibliografía.

2 Algunos de los textos tienen más de un tema, por cruce o contaminación, y en muchos casos son temas que no tienen relación con los que aquí consideramos.

3 En la mayoría de los casos el tema se da por el nombre conocido del romance español. 
Desde una perspectiva muy amplia el número de temas podría reducirse: por ejemplo, el tema de la muerte fingida en muchos casos se relaciona con el de la doncella raptada que escapa a su final trágico mediante esa argucia. Como no se trata de rastrear la presencia de tal o cual tema en una u otra tradición, sino de estudiar uno de los motivos que lo componen, el del entierro, hemos supeditado el manejo de los temas al motivo de nuestro interés.

\section{ELEMENTOS QUE CONTIENE EL MOTIVO DEL ENTIERRO}

Estrictamente, estos elementos son los siguientes: lugar del entierro, descripción del entierro y señales colocadas en la tumba. Sin embargo el motivo va, por lo general, acompañado de dos secuencias, una previa y una posterior, que es conveniente considerar cuando se estudia el motivo central, ya que están estrechamente ligadas a él o a alguno de sus elementos. Estas secuencias serían el momento de la muerte y la reacción de los vivos ante las señales de la tumba. Debido a la capacidad de condensación narrativa de la literatura tradicional, no todos los textos tienen estas secuencias complementarias. Por otra parte, también pueden faltar algunos de los elementos que hemos considerado como constituyentes del motivo del entierro. Veamos con más detalle estos elementos.

\section{El momento de la muerte}

Este elemento es especialmente significativo en aquellas baladas en las cuales son dos los personajes que mueren. La expresión de este elemento es formulística, ya que se trata, por lo general, de una ubicación temporal y se recurre a los tópicos de la tradición oral:

La un mort a mitja nit l'altre al despuntar el clar

("Don Lluis de Montalbá", Milá, p. 87)

El cavall morirá a nit i jo a la dematinada

(“Don Joan i don Ramon", Milá, p. 92)

La bèla es mòrta a miejanuèit

e lo galant a punta d'alba

("Gojats que setz a maridar", Petit, p. 92)

Pero también se señala el momento de la muerte cuando se trata de un solo personaje:

Ta Nanette elle est morte un jour de vendredi

(“La Nanette", Roy, p. 298)

4 En las referencias se dará únicamente el título de la balada, el autor de la colección y la página. 


\section{El lugar del entierro}

Éste es un elemento del motivo más frecuente que el anterior y por lo general más significativo, ya que en muchos casos es central para el desarrollo del tema mismo de la canción. Tal es el caso de "La belle qui fait la morte" o de "L'onore salvato", ambas canciones sobre el tema de la muerte fingida.

Au jardin de son père

dessous la fleur de lis

("La belle qui fait la morte", Roy, p. 109)

Ant el giardin de so padre

s'a jé d'un bel arborin

i sutreruma la bela

a l'umbra del guizumin

(“L'onore salvato", Nigra, p. 309)

En ambos casos es importante la ubicación de la sepultura en el jardín del padre para que la doncella pueda ser rescatada. Esta ubicación sólo podría ser sustituida por otra que conservara la verosimilitud narrativa de un lugar conocido por el posible salvador. La precisión del lugar (bajo el lirio o el jazmín) está en un nivel narrativo más superficial, ya que corresponde al discurso tradicional y no a la fábula que se narra.

En otras ocasiones se señala el sitio del entierro porque va a sufrir una transformación, por lo general como manifestación del amor más allá de la muerte:

N'enterran la bèla jos l'aspic

e lo galant al pé de l'arbre; quand ne sièron al cap de l'an,

l'aspic e l'arbre s'embracèron.

("Gojats que setz a maridar", Petit, p. 92)

Indicar el lugar del entierro también sirve para señalar diferencias entre los que han muerto; ya sean éstas de condición, jerárquicas, sociales o de otro tipo.

el xivall l'enterrareu just al mig la seu establa

i m'enterrareu a mi en el vas de Sant en Jaume.

(“Don Joan i don Ramon", Milá, p. 92)

Uno lo entierran 'nel coro y otro 'nel pie del altar

("Conde Olinos", Pelayo, p. 204)

Supli-me al altar magiur e'l me cavalin an piassa

("Mal ferito", Nigra, p. 149)

En otras ocasiones, el lugar del entierro lo determina la causa de la muerte, que puede impedir que éste sea regular o aprobado por la religión: 
Males d'amor nao tem cura nems' enterram en sagrado, enterram-se'em campos verdes onde se pastora o gado.

("Morte do principe dom Afonso", Ferré, p. 35)

Pero también puede ser determinante el deseo del difunto de que su tumba quede a la vista de todos o fuera del camposanto:

Au chemin de Saint-Jacques

enterrez-nous tous deux

(“La Pernette”, Roy, p. 111)

Si me muero deste mal no me entierren en sagrado, fáganlo en praderío donde no pase ganado.

("El mal de amor", Pelayo, p. 254)

\section{La descripción del entierro}

Por lo general, incluye a quienes llevan el ataúd, normalmente cuatro. Este tópico es muy abundante en las versiones francesas, y frecuentemente son soldados:

par quatre-z' officiers de guerre

("Complainte de la mal peignée" y “Adieux du soldat à sa mie”, Roy, pp. 120 y 285)

No siempre la expresión de aquellos que llevan el ataúd es realista; a veces se prefiere la imagen poética, aunque irreal, de que los porteadores sean palomas:

Nous l'ferons porter en terre

par quat' pigeons ramiers,

("Par un beau clair de lune", Roy, p. 115)

Otro detalle que gusta a la tradición es el de las velas en el entierro, al grado de que muchas veces es el único que aparece y su mención es suficiente para dar la noticia de la muerte:

cun sinquanta e due torce a faziú la luminá

(“La sposa morta", Nigra, p. 120)

Su marido, mi señora, muerto ha quedado en la guerra

debajo de un pino verde túvele yo la candela ${ }^{5}$

("Las señas del esposo", Pelayo, p. 256)

Otras veces el detalle de los porteadores de luces sirve para dar idea de la magnificencia del entierro:

5 La expresión "tenerle la candela" también podría interpretarse como acompañar a alguien durante su enfermedad y no necesariamente como entierro. 
Puis y aura cent damoiselles

qui s'ront pour porter des chandelles.

("Complainte de la mal paignée", Roy, p. 120)

\section{Señales sobre la tumba}

En nuestra muestra de textos son el elemento más abundante. Estas señales pueden ser de dos tipos: aquellas que surgen de manera más o menos extraordinaria de la tumba y aquellas otras que son colocadas por quienes cumplen con las disposiciones del entierro. Veamos algunos ejemplos del primer tipo:

na cova da Clar'Aninas nasceu um verde rosal e na cova do escudeiro nasceu um pinheiral.

(“A noiva abandonada", Ferré, p. 92)

Su la tumba d'bel galant j'é nassü'n pumin graná su la tumba d'la bela j'é nassü na mandolá.

(“Le due tombe", Nigra, p. 125)

de l'un surt una coloma i de l'altre un colomar.

("Don Lluis de Montalbá", Milá, p. 87)

Estas señales son símbolo de los que han muerto, y representarán sus sentimientos en muchos de los casos; frecuentemente el motivo del entierro con señales que surgen de las tumbas corresponde al tema del amor más poderoso que la muerte.

Las señales que se colocan en la o las tumbas son generalmente armas, flores, árboles que se plantan, o una cruz, como en el romance de "Don Manuel”. He aquí algunos ejemplos:

Caperatz-me de ròsas e mon amic de flors

("Petita Margarida", Petit, p. 86)

A damunt hi posareu l'espasa desembainada,

("Don Joan i don Ramon", Milá, p. 92)

An sima a cula tumba piantran d'le roze e fiur.

("Fior di tomba", Nigra, p. 129)

Par la mitan d'la fosse un romarin planté

(“Par un beau clair de lune”, Roy, p. 115)

El motivo del entierro se puede prolongar, como ya hemos dicho, con una secuencia relacionada con las señales en la tumba. Cuando son señales aparecidas, que expresan por lo general las relaciones entre los muertos, también están sometidas a vicisitudes y pueden tener diversos avatares: 
A rainha, como inveja, logo los mandou cortar, mas os rebentos que ficaram tornaram a rebentar. (“A noiva abandonada", Ferré, p. 92)

En el caso de señales colocadas, pueden provocar en el que pasa y las ve un sentimiento compasivo o una oración por el difunto que ha perdido la vida a causa del amor o de la guerra:

diseràn los passejants: de qué son aquelas armas?

De l'enfant de Lisardon qu'èra estat a las batalhas.

(“A las batalhas del Rei", Petit, p. 98)

Tous les passants qui passent

n'en prendront une fleur

et prieront Dieu qu'il fasse

grâce à deux amoureux.

(“La Pernette", Roy, p. 111)

Cómo aparece el motivo en el texto

La extensión del motivo del entierro en el texto es muy variable y está relacionada con el número de elementos que contiene. Puede aparecer en forma condensada y explícita:

y aquí se enterró Don Pedro la prenda que más querías

("La muerte ocultada", Pelayo, p. 234)

También se puede expresar en formas muy extensas, con múltiples elementos, llenos de detalles, como en el caso de la versión francesa de "Mambrú", en la cual se describen los cuatro oficiales que entierran a Mambrú, las armas que llevan, y se da además el detalle de los cuatro romeros que se plantan en las esquinas de la tumba, en cuya rama más alta canta un ruiseñor. Un caso similar al anterior es el de "Par un beau clair de lune".

Como ya hemos dicho, hay expresiones del entierro que podríamos llamar indirectas, puesto que se limitan a señalar uno solo de los elementos del motivo, por ejemplo la vela o las luces que acompañan a los entierros. Tal es el caso de "La sposa morta", "La prova" y "Las señas del esposo"

En la cadena narrativa de las distintas baladas, el motivo del entierro puede asumir funciones diversas: en algunos casos es una noticia que le comunica un personaje a otro, en otros es la disposición que da un personaje sobre su propio entierro y en otros más es información que proporciona el narrador.

Al ser el entierro un motivo trágico, por lo general se encuentra en la secuencia conclusiva del texto, y muchas veces la reacción de los que pasan ante la tumba es el cierre de la narración.

A continuación, y en forma condensada, se muestra cómo aparece el motivo del entierro en las baladas que forman nuestro corpus, especificando si es el narrador o 
el personaje el que nos lo dice, si aparece en el interior del texto o en su conclusión y si se nos da como información, noticia o disposición testamentaria. Distinguimos los datos proporcionados por un personaje a otro (noticias) de los datos proporcionados al lector o al escucha (informes):

Personaje, conclusión, disposiciones: 11 baladas.

Personaje, conclusión, informes: 4 baladas.

Personaje, conclusión, noticias: 1 balada.

Personaje, interior, disposiciones: 1 balada.

Personaje, interior, informes: 3 baladas.

Personaje, interior, noticias: 9 baladas.

Narrador, conclusión, informes: 6 baladas.

Narrador, interior, informes: 2 baladas.

Cuando el entierro contiene disposiciones testamentarias por lo general es el motivo principal de la balada. Por el contrario, cuando contiene noticias tiende a ser un motivo secundario (aunque en muchos casos tiene importancia, ya que es el motivo generador de las acciones pues el conocimiento del entierro de alguien, y por lo tanto de su muerte, precipita la acción central de la narración). Así ocurre en las baladas con el tema de la aparición de la amada difunta.

\section{EL MOTIVO DEL ENTIERRO EN UN MISMO TEMA BALADISTICO}

En las distintas tradiciones encontramos el mismo tema baladístico y en ocasiones el motivo del entierro tiene una expresión similar, por ejemplo en las señas del esposo:

Debajo de un pino verde túvele yo la candela.

(“Señas del esposo", Pelayo, p. 256)

Cum sinquanta torce avische lo portavo a suterá.

(“La prova", Nigra, p. 315)

En ambos textos el motivo se ha reducido a su mínima expresión y se apoya en el elemento lumínico que acompaña al entierro. También en los dos casos es el personaje el que da noticias de la muerte, y tiene por objeto el probar la fidelidad de la esposa.

También puede darse el caso de que el motivo tenga similitud de funciones, colocación y objetivos y sin embargo se desarrolle con distinta amplitud y tomando en cuenta distintos elementos. Por ejemplo, las baladas sobre el tema de la amada difunta:

Tua amada já morreu já morreu qu'eu bem a vi, quatro condes a levaram p'r'á capela de Bonfim.

(“O soldado", Ferré, p. 88) 
Que el dia del seu enterro jo la missa vaig a oir, les cortines del palacio jo de dol les vaig cobrir, els infants que ella tenia jo de dol los vaig vestir.

("La comtessa morta", Milá, p. 111)

Vostra spuzzetta l'é andá a la ceza a la ceza ben cumpagná cun sinquanta e due torce a faziú la luminá.

("La sposa morta", Nigra, p. 120)

son corps il est en terre

("La Nanette", Roy, p. 111)

En esta balada el motivo está en una posición interna en el texto y su función es motora, puis genera las acciones principales. La expresión en cada texto es bastante diferente tanto en su extensión como en los motivos que contiene.

\section{Expresiones similares del motivo en distintos temas}

También se puede encontrar el caso contrario, esto es, el motivo expresado en forma similar pero con funciones diferentes en distintos temas. Un ejemplo de esto sería el del llamado "comodín romancístico"\% del entierro fuera de sagrado. Este motivo, expresado en forma bastante parecida, aunque con variantes significativas de sentido, lo encontramos en textos de tema tan distinto como "Morte do principe dom Afonso de Portugal", "O veneno de Moriana", "Mal de amor" y "Don Manuel". La función del motivo en cada uno de los textos es distinta; sin embargo, por la propia expresión tiene una serie de características constantes: el estar en posición conclusiva en el texto, el ser una disposición testamentaria, etcétera. Desde luego que el sentido del motivo será muy diferente si corresponde a una muerte violenta o a muerte por amor, por ejemplo:

Enterram-s'em campos verdes onde se pastora o gado, c'uma maozinha de fora c'o sê letreiro armado, ("Morte do principe dom Afonso", Ferré, p. 36)

Laurisberta, s'ê morrer, enterra-m'em lugar sagrado, enterra-me'm campos verdes onde se pastora o gado, (“O veneno de Moriana", Ferré, p. 184)

Si me muero deste mal, no me entierren en sagrado; fáganlo en un praderío donde non pase ganado; ("Mal de amor", Pelayo, p. 254)

Hay otros casos de motivos viajeros menos extremos que el de este comodín; por

- Diego Catalán usa este término en forma acertada, a mi ver, en Por campos del Romancero, Madrid, Gredos, 1970, p. 144. 
ejemplo, el que contiene el elemento de las dos tumbas. En primer lugar lo encontramos en baladas de muerte por amores contrariados:

Nos faretz una tomba per nos botar tots dos.

Caperatz-me de ròsas e mon amic de flors.

Los qui angan a Sant Jaumes pregaràn a Dieu per nos!

("Petita Margarida", Petit, p. 86)

Expresión muy similar la encontramos en la balada francesa del mismo asunto, "La Pernette", pero también la encontramos en baladas cuyo tema es la muerte del soldado o del caballero que regresa de una ausencia prolongada, en cuyo caso las dos tumbas son para el guerrero y su caballo. Así ocurre en "Don Peire i don Joan", "Don Joan i don Ramon", "A las batalhas del Rei" y "Mal ferito".

Supli-me a l'autar magiur e' me cavalin in piassa o crubi-me d'roze e fiur e'l me cavalin de giolifrada.

Tuta la gent ch'a passeran a diran: che gran dalmage.

("Mal ferito", Nigra, p. 149)

Como se ve por el ejemplo anterior hay similitud de expresión, pero diversidad de sentido. Otro tema en el cual podemos encontrar este element o de las d os tumbas en el motivo del entierro es, por ejemplo, el de "la pesca del anillo", en el cual normalmente muere quien hace el rescate, aunque en el ejemplo italiano muere la mujer. Este motivo de las dos tumbas está ligado al de las señales en las tumbas que sufren transformaciones después de un tiempo. Es frecuente el paso de un motivo a otro, perdiéndose en el segundo el sentido de la disposición testamentaria original.

Como se ha podido ver en esta cala, son muchos los temas que se pueden analizar en un estudió comparativo de baladas románicas. Aquí simplemente hemos señalado la importancia que puede tener en un estudio de este tipo la descripción de los distintos elementos que integran un motivo, las funciones que puede desempeñar éste dependiendo de su colocación en la secuencia narrativa o de la yoz que lo expresa (será noticia o información, según sea el personaje o el narrador quien lo diga). Una noticia en posición interna tiene un sentido distinto de una noticia en posición conclusiva; en el primer caso se tratará por lo general de un motivo secundario y en el otro posiblemente sea parte del tema central. También es importante recordar la elasticidad que tiene un mismo motivo para integrarse a temas distintos, así como la permanencia de ciertas expresiones formulísticas comunes o la preferencia de una tradición por alguna de ellas.

Después de haber hecho esta cala parece claro que el funcionamiento de la gran tradición baladística románica es completamente similar y tiene los mismos recursos que las distintas tradiciones en forma individual, y que en la misma forma debe funcionar la balada románica dentro de la baladística europea. 
APENDICE: TEXTOS

Le due tombe

Ant ël bosco di Cazale bela fia va cantá.

-O canté, canté, fieta, fín che sei da maridá!

Bel galant va da so pare: - Vostra fia m'la voli dá?

So pare j'a fait risposta ch'a i la vuria nen dá.

Bel galant l'é vnü malavi e la bela a na stá mal.

Bel galant viv a panade e la bela a pan gratá.

Bel galant l'é mort a l'alba e la bela a sul levá.

Bel galant l'an sutrá an ceza e la bela sul piassal.

Su la tumba d'bel galantj'e nassủ'n pumin graná.

Su la tumba de la bela j'é nassü na mandolá.

Tanto bin cum a crëssio, fazzio umbra a tres sitá, Alessandria e Valensa e la pi bela Cazal.

(Nigra, p. 125)

(En el bosque de Casale una bella muchacha va a cantar./ Oh, canta, canta, jovencita, mientras eres casadera./ Bello galán va con su padre: ¿quiere darme a su hija?/ El padre le responde que no se la quiere dar./ Bello galán cae enfermo y la bella está mala./ Bello galán vive de empanadas y la bella de pan molido./ Bello galán ha muerto al alba y la bella al salir el sol./ Al bello galán lo han enterrado en la iglesia y a la bella en la plaza./ Sobre la tumba del bello galán ha nacido un granado/ y sobre la tumba de la bella ha nacido un almendro./ Tan bien como crecieron daban sombra a tres ciudades,/ Alessandria y Valenza y la más bella Casale.)

\section{Mal ferito}

O s’a i sun tre giügadur ch'a n' in giögo de le carte.

L’àn giögà e stragiöga, e poi si taco a parole;

e da parole a cutei, e da cutei a pistole.

El prim culp che lur l'àn fáit, l'àn feri lo ruè dla Spagna.

Gentil galand munta a caval, per andar a la sua caza.

Sua mama à l’à vist rivar, cun ủn’ária cozl pázia.

-O maman, pruntè-me un let, ün let di piüma d'oca;

e i ninsolin di téila d'lin, e la querta di verdüra.

A mezanóit che mi sun mort e'l me cavalin ant l'alba.

Süpli-me a l'autar magiur, e'l me cavalin an piassa.

O crübi-me d'roze e fiur e'l me cavalin d'giolifrada.

rüta la gent ch'a passeran, a diran: -che grant dalmagi! 
Che dalmagi del cavalin e ancur pi dlo ruè dla Spagna!

(Nigra, p. 149)

(Son tres jugadores que juegan a las cartas./ Han jugado y rejugado y después han pasado a las palabras,/ de las palabras a los cuchillos y de los cuchillos a las pistolas./ El primer golpe que han dado ha herido al rey de España./ E1 gentil galán monta a caballo para ir a su casa./ Su madre lo ha visto llegar con aire abatido./ -Oh, madre, prepárame un lecho, un lecho de plumas de oca,/ con sábanas de lino y la colcha de verdor./ A medianoche yo he muerto y mi caballito antes del alba./ Entiérrenme en el altar mayor y a mi caballito en la plaza,/ cúbranme de rosas y flores y a mi caballito de claveles./ Toda la gente que pasará dirá ¡qué desgracia!/ ¡Qué desgracia del caballito! y aún más del rey de España!)

Don Joan i don Ramon

Don Joan i don Ramon vienen de la cacada i com foren en el camp l'afinaren de llançades.

Don Joan romangué mort Don Ramon poc li faltava, so mare al veure'l venir baix de la finestra estava:

-D'on veniu, el meu fillet, d'on veniu, la color mudada?

- Mare, non digueu aixó, vos mateixa me'n sou causa.

- Pujau, don Ramon, pujau a damunt la cambra alta

i vereu vostra muller que n'ha parit una infanta.

-No m'alegra la muller ni tampoc la meua infanta,

perque non la veuré gran en el camp a portar llança.

Entre jo i mon cavall portam vint-i-nou llançades:

el xivall porta les nou trist de mi porto les altres.

El cavall morira à nit i jo a la dematinada;

el xivall l'enterrareu just al mig la seu establa

i m'enterrareu a mi en el vas de Sant en Jaume.

A damunt hi posareu l'espasa desembainada,

si demanen qui m'ha mort: don Joan a la caçada.

(Milá, p. 92)

(Don Juan y don Ramón venían de la caza/ y como estaban en el campo se enfrentaron a lanzadas./ Don Juan quedó muerto, a don Ramón poco le faltaba./ Su madre al verlo venir bajo de la ventana estaba. ¿ ¿De dónde viene mi hijo, de dónde viene con la color mudada?/ Madre, no diga eso, métame en su casa./ Subid, don Ramón, subid a la cámara alta/ y veréis a vuestra mujer que ha parido una infanta./ No me alegra la mujer ni tampoco la mi infanta/ porque no la veré en el campo al portar lanza./ Entre mi caballo y yo traemos veintinueve lanzadas;/ el caballo trae las nueve, triste de mí, yo traigo las otras./ El caballo morirá en la noche, yo al amanecer./ Al caballo lo enterraréis justo en medio de su establo, / a mí me enterraréis abajo de San Jaume/ y encima pondréis la espada desenvainada./ Si preguntan quién me ha muerto: don Juan en la caza.) 
Petita Margarida

-Petita Margarida, quin marit volètz-vos?

Volètz lo filh d'un comte o lo filh d'un baron?

-Non vòli pas de comte ni tanpauc de baron:

vòli mon amic Pèire, lo qu'es dins la preson.

- Petita Margarida, Pèire n'es pas per vos;

Pèire es jutjat a pendre deman, al punt del jorn.

-Monsenh, si penjatz Pèire, penjatz nos a tots dos.

Nos faretz una tomba per nos botar tots dos.

Caperatz-me de ròsas e mon amic de flors.

Los qui angan a Sant Jaumes pregaràn Dieu per nos!

(Petit, p. 86)

(Pequeña Margarita, ¿qué marido quieres?/ ¿Quieres al hijo de un conde o al hijo de un barón?/ Yo no quiero conde ni tampoco un barón:/ yo quiero a mi amigo Pedro que está en la prisión./ Pequeña Margarita, Pedro no es para ti,/ Pedro ha sido condenado a ser colgado mañana al despuntar el día./ Mi señor, si colgáis a Pedro colgadnos a los dos./ Hacednos una tumba para echarnos a los dos./ Cubridme de rosas y a mi amigo de flores./ Los que van a San Jaime rogarán a Dios por nosotros.)

\section{La Pernette}

La Pernette se lève deux heur's avant le jour et prend sa quenouillette son joli petit tour.

A chaque tour qu'elle file elle pleure en dessous. Sa mère lui demande -Pernette qu'avez-vous? Avez-vous mal à la tete? ou bien le mal d'amour? - Je n'ai pas mal de tête, mais bien le mal d'amour. -Ne pleure pas, Pernette, nous te marierons avec le fils d'un prince ou celui d'un baron.

- Je ne vequx pas de prince ni le fils d'un baron; je veux mon ami Pierre 
qui est dans la prison.

-Tu n'auras pas ton Pierre

ou bien nous le pendrons.

-Ah, si vous pendez Pierre

pendez-moi donc aussi.

Au chemin de Saint-Jacques

enterrez-nous tous deux, couvrez Pierre de roses

et moi de mille fleurs.

Les pèlerins qui passent

prieront Dieu pour nous deux.

Tous les passants qui passent

n'en prendront une fleur

et prieront Dieu qu'il fasse

grâce à deux amoureux.

(Roy, p. 111)

(La Pernette se levanta/ dos horas antes del alba/ y toma su pequeña rueca/ su gracioso y pequeño giro./ A cada giro del hilo/ ella llora sobre él./ Su madre le pregunta:/ Pernette, ¿qué es lo que tienes?/ ¿Te duele la cabeza/ o tienes mal de amores?/ No me duele la cabeza/ pero tengo mal de amores./ No llores más, Pernette,/ nosotros te casaremos/ con el hijo de un príncipe/ o el de un barón./ Yo no quiero al príncipe/ ni al hijo de un barón,/ yo quiero a mi amigo Pierre/ que está en la prisión./ Tú no tendrás a tu Pierre/ o bien nosotros lo colgaremos./ Ah, si ustedes cuelgan a Pierre/ cuélguenme a mí también./ En el camino de Santiago/ entiérrennos a los dos./ Cubran a Pierre de rosas/ y a mí de mil flores./ Los peregrinos que pasen/ rogarán a Dios por nosotros;/todos los caminantes que pasen/ cogerán una flor/ y pedirán a Dios que/ perdone a dos amantes.)

\section{A noiva abandonada}

-Escudeịro, nã namores a filha do tž senhor, qu'há-de te meter em conta morreres por teu amor. Escudeiro assim que soube, longes terras foi casar, Aninas louca d'amor lo foi buscar.

Nẩ perguntou por igreja nem per santa do altar, perguntou por escudeiro sua terra natural.

-O escudeiro nã 'tá; foi para caça caçar.

- Se năo é com muita pressa

poi se o manda chamar, ou por a filha Inês ou por a cunhada Guiomar.

-Não era com muita pressa mas também não é devagar, qu'ê deixei mê pai na mesa, lá por horas de jantar. 
Deixei o jarro com água e o vinho a refinar.

-O que fazes Don'Aninas, de minha terra natural?

-É os amores do escudeiro que por 'qui me faz andar.

- Também os vossos, senhora, me fizeram desterrar.

Dá-me licença, senhora, par'um abraco le dar?

-Dá-le um, ou dá-le dois,

mas s'isso é mal d'amores como há-d'ela tornar?

Morreu um e morreu outro, ambos foram a enterrar;

na cova da Clar'Aninas nasceu um verde rosal

e na cova do escudeiro nasceu um pinheiral.

Cresceu um e cresceu outro qu'ó céu s'iam juntar

e todas as aves do mundo lá iam repousar.

A rainha, com inveja, logo los mandou cortar, mas os rebentos que ficaram tornaram a rebentar.

Cresceu um e cresceu outro qu'o céu se foram juntar.

(Ferré, p. 92)

(Escudero, no enamares a la hija de tu señor,/ que si se ha de dar cuenta moririas por tu amor./ El escudero que así lo supo a lejas tierras se fue a casar,/ Aninas, loca de amor, lo fue a buscar./ No preguntó por iglesia ni por santa del altar,/ preguntó por el escudero de su tierra natural./ - El escudero no está, fue a la caza a cazar./ -Si no tiene mucha prisa se manda buscar,/ por la hija Inés o la cuñada Guiomar./ -No con mucha prisa, pero tampoco despacio,/ que dejé a mi padre a la mesa a la hora de comer./ Dejé el jarro con agua y el vino a refinar./ ¿Oh, qué haces, Don'Aninas, de mi tierra natural? / -Son los amores del escudero que por aquí me hacen andar./ - También los vuestros, señora, me hicieron desterrar./ ¿Me da licencia, señora, para un abrazo le dar?/ Dale uno, o dale dos, / ipero si tiene mal de amores cómo ha de tornar a ella?/ Muere el uno, muere el otro, ambos fueran a enterrar;/ en la tumba de Clar'Aninas nació un verde rosal/ y en la tumba del escudero nació un pinar./ Crece uno y crece el otro que se iban a juntar/y todas las aves del mundo a hí se iban a posar./ La reina con envidia, luego los mandó cortar, / pero los vástagos que quedaron volvieron a renovar./ Crece uno y crece el otro que después se fueron a juntar.)

\section{Conde Olinos}

¿Quién se dol del conde Olinos, que niño pasara el mar!

Lleva su caballo al agua una noche de lunar;

mientras el caballo bebe, él le canta este cantar:

-Bebe, mi caballo, bebe, Dios te me libre de mal, de los peligros del mundo y de las ondas del mar; de los castillos de Arriba que me quieren mucho mal. La reina mora lo oyera de altas torres donde está:

-Escuchadle, mis doncellas, las que dormís, recordad, 
y oiredes a la serena como canta por el mar.

Respondió la más chiquita, ¡más le valiera callar!

- Aquella no es la serena, nin tampoco su cantar, aquel es el conde Olinos que conmigo va a casar.

La reina que aquello oyera, ambos los mandó matar.

Uno lo entierran 'n el coro, y otro 'n el pie del altar.

D'ella nació verde oliva, d'él nació verde olivar.

Crece el uno, crece el otro, ambos iban a la par; cuando hacía aire d'arriba ambos se iban a abrazar; cuando hacía aire d'abajo ambos se iban a besar. La reina que aquello ve ambos los manda cortar. D'ella naciera una fuente d'él nació río caudal.

"Quien tuviera mal de amores aquí se venga a bañar."

La reina que aquello oyera también se fuera a lavar. -Detente, reina, detente, no me vengas dexobar, cuando yo era Blanca Flor tú me mandaste matar, cuando yo era verde oliva, tú me mandaste cortar; ahora soy fuente clara, non me puedes facer mal; para todos he de correr para ti me he de secar.

(Pelayo, p. 204)

\section{BIBLIOGRAFIA}

FERRE, Pere, Romances tradicionais. Funchal, Cam. Municipal de Funchal, 1982.

MENENDEZ PELAYO, Marcelino, Antología de poetas líricos castellanos, t. VII. Buenos Aires, Espasa-Caple, 1952.

MILÁ, Manuel, Romancer Cataló. Ed. J. A. Paloma, Barcelona, Edicions 62/“La Caixa”, 1980.

NIGRA, Constantino, Canti popolari del Piemonte. Torino, Frassati, 1888.

PETIT, Jean-Marie y Jean TENA, Romancero occitan. Paris, Maspero, 1971.

ROY, Claude, Trésor de la poésie populaire. Paris, Seghers, 1954. 